\title{
Method of Cognitive Semantic Analysis of Russian Sentence
}

\author{
Alexander Bolkhovityanov and Andrey Chepovskiy \\ Higher School of Economics, \\ Moscow, Russia \\ alexander.bolkhovityanov@gmail.com, \\ achepovskiy@hse.ru
}

\begin{abstract}
In this paper, we propose two mathematical models intended for analyzing the Russian sentence to detect noun phrases and participial clauses. Considered algorithms designed on the basis of the proposed models can improve procedure of syntactic parsing. Algorithm for participial clause identification is based on the concept of syntactic relation between verb and dependent syntactic units in the Russian language.
\end{abstract}

\section{Introduction}

The main task of semantic analysis is creating representation of a text, allowing to correlate the information contained in the text with the knowledge system. The goal of cognitive semantic analysis [1] is formation of such intermediate representation. The creation of mathematical models which help to solve engineering problems of text processing requires to solve the problem of cognitive modeling of a text.

This problem well studied for languages with a simple syntactic structure, for example English. But it is a complicated task for languages which have more complex structure, for example Russian. There are a lot of syntactic relations in the Russian sentence.

The information about the syntactic structure of the analyzed text requires formation of the intermediate representation. The result of parsing is represented as a syntactic dependency tree [2], which can be obtained on the basis of syntactic relations among the constituent elements of the text. Thus, we are faced with the task of establishing syntactic relations among syntactic units of sentence.

In this paper we propose mathematical models and algorithms, which help us to solve the problem of identifying syntactic relationships among the elements of the text completely but in some acceptable approximation for the real systems of the text processing.

We describe mathematical models for noun phrase and participial clause identification. In Section 2 we propose mathematical models and algorithms for noun phrase and participial clause identification. We note that participial clause identification is based on the concept of syntactic relations between verb and dependent syntactic units, noun phrases for example. 


\section{Mathematical Models}

In this section, we provide mathematical models and algorithms for noun phrase and participial clause identification in the context of the Russian sentence.

Let us introduce some auxiliary sets which needed for formal definition of the following algorithms. These sets are:

1. Set of parts of speech in Russian PoS $=\{N O U N, A D J, V E R B, C N U M$, $P A R T\}$, where $N O U N$ - noun, $A D J$ - adjective, $V E R B$ - verb, $C N U M$ - cardinal number, PART — participle.

2. Set of grammar cases in Russian $G C=\{N, G, D, A, I, P\}$, where $N$ nominative case, $G$ - genitive case, $D$ - dative case, $A$ - accusative case, $I$ - instrumentative case, $P$ - preposition case.

3. Set of genders in Russian Genders $=\{M, F, N\}$, where $M$ - masculine gender, $F$ - feminine gender, $N$ - neuter gender.

4. Set of numbers in Russian Numbers $=\{S G, P L\}$, where $S G$ - singular, $P L$ - plural.

The word $w$ may be represented as a list of the results of morphological analysis, grouped by parts of speech. $M A(w)$ function sets the correspondence between the set of words and results of morphological analysis mentioned above:

$$
M A: w \rightarrow\left(G_{1}, \ldots, G_{n}\right)
$$

where $G_{i}=\left(G I_{1}, \ldots, G I_{k}\right), G I_{j}=$ (pos, gc, gender, number $)$, pos $\in$ PoS, gc $\in$ $G C$, gender $\in$ Genders, number $\in$ Numbers, $\forall j \in[1, k-1]:$ pos $_{j}=$ pos $_{j+1}$. $G I_{j}$ is a particular result of morphological analysis of the word $w$. There are more groups of morphology analysis $G_{i}$ because of the homonymy in the Russian language. Thus, the morphology analysis results may be represented in the unbundled form: $M A(w)=\left(G I_{1}, \ldots, G I_{p}\right)$. Further, we use the results of $M A(w)$ above mentioned.

\subsection{Noun Phrase Identification}

Let us introduce some auxiliary notations.

Subordinate syntactic relation is a pair where the first element of a pair $w$ is a word and the second element $R L(w)$ is a list of subordinate words: $r_{i} \in R, r_{i}=$ $(w, R L(w)), R L(w)=\left(w_{1}, \ldots, w_{l}\right)$.

$R$ is a set of subordinate syntactic relations. At a preliminary step one may add subordinate syntactic relation with empty relations list: $\forall w: R=R \cup(w,())$ where () means empty list for each word $w$ in the sentence.

The input of the algorithm is a sentence composed of words Sent $=w_{1}, \ldots, w_{n}$, where $w_{i} \in \Sigma^{+}$, and $\Sigma$ is the Russian alphabet. The algorithm consists of three basic steps:

1. Setting subordinate syntactic relations in a pair (lw,rw), where $l w$ is a left word, $r w$ is a right word in a pair. For example, if we have an input sentence Sent consisting of the sequence of the words $w_{1}, w_{2}, \ldots, w_{n}$, then we can consider the pair $\left(w_{1}, w_{2}\right)$, where $l w=w_{1}, r w=w_{2}$. 
2. Setting syntactic relations inside homogeneous parts of the sentence;

3. Identifying of the noun phrases.

Relations are set in the pair $(l w, r w)$. Next, we propose conditions under which we add the corresponding relation to the relations set $R$.

Here are the basic steps of the algorithm.

\section{Setting subordinate syntactic relations}

1. If $\exists G I_{i} \in M A(l w):$ pos $_{i}=N O U N$ and $\exists G I_{j} \in M A(r w): p o s_{j}=N O U N$ and $g c_{j}=G$ then add relation for word $l w: R L(l w)=R L(l w) \cup(r w)$;

2. If $\exists G I_{i} \in M A(l w): \operatorname{pos}_{i}=A D J$ and $\exists G I_{j} \in M A(r w): p o s_{j}=N O U N$ and $g c_{i}=g c_{j}$, gen $_{i}=g_{e n}$ and either num $_{i}=$ num $_{j}$ or num $_{i}=S G$ then add relation for word $r w: R L(r w)=R L(r w) \cup(l w)$;

3. If $\exists G I_{i} \in M A(l w): \operatorname{pos}_{i}=C N U M$ and $\exists G I_{j} \in M A(r w): p o s_{j}=N O U N$ and $g c_{j}=G$ and either $g c_{i}=N$ or $g c_{i}=G$ then add relation for word $l w$ : $R L(l w)=R L(l w) \cup(r w)$.

Setting syntactic relations inside homogeneous parts of the sentence. Let us introduce set of copulas $C$ by which homogeneous parts of the sentence are separated. We form the list of homogeneous nouns $S=\left\{s_{1}, \ldots, s_{m}\right\}$ in accordance with following conditions:

1. Nouns are separated with copula $c_{i} \in C$;

2. $\forall s_{i} \in S, \exists s_{j} \in S: g c_{i}=g c_{j}$.

The algorithm is the sequential changing of subordinate syntactic relations for nouns from homogeneous parts of the sentence.

If two last nouns $s_{n-1}, s_{n} \in S$ are separated with "И" and $\exists s_{l} \in R L\left(s_{n}\right)$ : $\exists G I_{i} \in M A\left(s_{l}\right): \operatorname{pos}_{i}=N O U N$ then if $\forall s_{k} \in R L\left(s_{n-1}\right), \neg \exists G I_{i} \in M A\left(s_{k}\right)$ : pos $_{i}=N O U N$ then $r_{n-1}=\left(s_{n-1}, R L\left(s_{n-1}\right) \cup s_{l}\right)$. If $\exists G I_{i} \in M A\left(s_{k}\right):$ pos $_{i}=$ $N O U N$ then one should finish this part of the second step and perform next check.

If $\forall s_{i} \in S, \exists G I_{j} \in M A\left(s_{i}\right): g c_{j}=G$ and if $\exists r_{p} \in R: s_{1} \in R L\left(w_{p}\right)$ and $\exists G I_{j} \in M A\left(w_{p}\right): \operatorname{pos}_{j}=N O U N$ then $\forall r_{q}=\left(w_{q}, R L\left(w_{q}\right)\right.$ if $s_{1} \in R L\left(w_{q}\right)$ then $R L\left(w_{q}\right)=R L\left(w_{q}\right) \cup\left(s_{2}, \ldots, s_{m}\right)$

Noun phrase identification. We composite an undirected graph $G_{R}=(V, E), E=$ $E_{q} \cup E_{u}$ on the basis of relations from set $R$. The vertices in this graph are the sentence words, the edges are the subordinate syntactic relation. There are two types of edges: u-edges and q-edges. If $e_{q_{l}}=\left(v_{i}, v_{j}\right)$ then $\exists r_{p} \in R: v_{j} \in R L\left(v_{i}\right)$ and if $e_{u_{l}}=\left(v_{i}, v_{j}\right)$ then $\exists r_{p} \in R: v_{j} \in R L\left(v_{i}\right), \operatorname{pos}_{v_{j}}=A D J$. See Figure 1 for example.

Noun phrase can be identified by using graph $G_{R}$. The path $p=\left(v_{1}, \ldots, v_{k}\right)$ in the graph $G_{R}$ corresponds to the noun phrase if met one of the following conditions:

1. if $k=2$ then $e=\left(v_{1}, v_{2}\right), e \in E_{q}$;

2. if $k>2$ then $\exists e_{i} \in E_{u}$ and $\exists e_{j} \in E_{q}$.

Identified noun phrases form the set $N P h=\left\{p_{1}, \ldots, p_{f}\right\}$ 


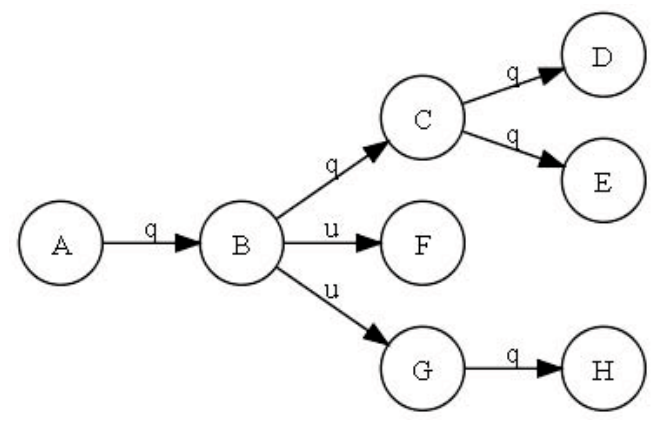

Fig. 1. Example of the relations graph

\subsection{Participial Clause Identification}

The algorithm for participial clauses identification works with the set of noun phrases NPh identified with the algorithm which was described in previous section. Grammatical information for noun phrase is determined by the main word of the noun phrase. The algorithm is based on the assumption that participle has the same model of relations with dependent syntactic units as verb from which it is formed. Let us introduce some definitions.

Definition 1. Restriction is a tuple $r=($ preposition, gc, type) where preposition defines preposition with which the verb is used in a sentence, gc defines grammar case of the dependence noun phrase, type defines the type of the dependence noun phrase, which may be free, connected or conditioned.

Definition 2. Relation between verb and dependent syntactic units is a map that assigns to each verb in the initial form a list of restrictions:

$$
V R: v \rightarrow\left(r_{1}, \ldots, r_{n}\right)
$$

The verb restrictions dictionary has been obtained from the dictionary [5] with some auxiliary modifications.

There are restrictions for some types of participles, for example the passive participles that supplement the list of restrictions obtained from the participle derived from the verb.

Thus, we can introduce the concept of the relation between participle and dependent syntactic units in the Russian sentence.

Definition 3. Relation between participle and dependent syntactic units is a map that assigns to each participle a list of restrictions:

$$
P R: p \rightarrow\left(r_{1}, \ldots, r_{k}\right)
$$

The restrictions for the participle $p$ are obtained from the verb $v$ restrictions and the additional participle restrictions $r_{1}, \ldots, r_{l}$ :

$$
P R(p)=V R(v) \cup\left(r_{1}, \ldots, r_{l}\right)
$$


The participial clause is the participle $p$ and dependent noun phrase $n p h \in$ $N P h$ which conforms one of the restrictions from the list $P R(p)$.

Thus, we may formulate the algorithm for participial clause identification: $\exists w_{i}: \exists G I_{j} \in M A\left(w_{i}\right): \operatorname{pos}_{j}=P A R T$ and $\exists r_{p} \in P R\left(w_{i}\right)$ and $\exists n_{q} \in N G$ : prep $_{p} \in n_{q}, g c_{p}=g c_{q}$. Noun phrase $n_{q}$ and participle $w_{i}$ make the participial clause.

\section{$3 \quad$ Evaluation and Example}

For evaluation we use the texts from the news corpora on the political topics. The experiments show that the precision of the noun phrases identification is about $95 \%$. In 3 is shown that for the Russian scientific texts precision is about $88 \%$. Own system for noun phrase identification was developed in AOT project. In their paper 4] the authors describe the templates in the regular language. This formalism has some disadvantages. For example, it is impossible to describe the discontinuous noun phrases with the proposed regular language. We note, that our system is devoid of such shortcomings and it can analyze sentences any level of complexity.

The precision of the participial clause identification is about $98 \%$ and this is consistent with the results of our preliminary theoretical analysis.

Let us give an example of noun phrase identification algorithm. We have sentence: Оценки состояния и перспектив российской экономики. Denote words in sentence by letters: Оценки - A, состояния - B, перспектив - D, российской - X, экономики - C. In Figure 2 you can see syntactic relations graph between words in sentence.

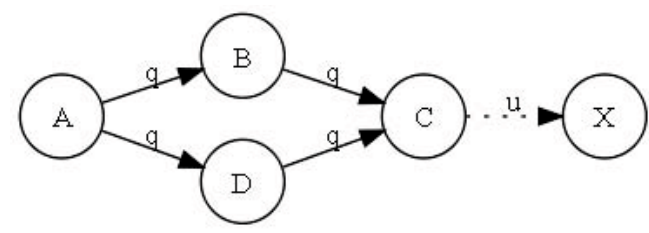

Fig. 2. Relations graph for sentence from example

As a result of the algorithm we found the following noun phrases (words is in the canonical form):

1. экономика российский;

2. перспектива экономика российский;

3. состояние экономика российский;

4. оценка перспектива;

5. оценка состояние;

6. оценка состояние экономика; 
7. оценка перспектива экономика;

8. оценка состояние экономика российский;

9. оценка перспектива экономика российский.

\section{Conclusion}

In this paper we examined the mathematical models designed to solve a broad class of problems on cognitive analysis of text information. The algorithms, formulated in the terms of the proposed models can efficiently and with high accuracy detect dependent syntactical constructions that help us to realize the efficient algorithm of syntactical analysis of the Russian texts. This is achieved by reducing the large number of possible links in the graph arising after morphology analysis due to homonymy in the Russian language. This reduction is possible when we form chains from word combination, preposition and information about relations between verb or participle and dependent syntactic unit.

\section{References}

1. Croft, W., Cruse, D.A.: Cognitive linguistics (Cambridge Textbooks in Linguistics). Cambridge University Press, Cambridge (2004)

2. Jurafsky, D., Martin, J.H.: Speech and Language Processing: An Introduction to Natural Language Processing, Computational Linguistics, and Speech Recognition, 2nd edn. Prentice Hall, New Jersey (2008)

3. Belonogov, G.G., Kuznetsov, B.A.: Yazykovye sredstva avtomatizirovannyh informatsionnyh sistem (in russian). Nauka, Moscow (1983)

4. Pankratov, D.V., Gershenzon, L.M., Nozhov, I.M.: Opisanie fragmentacii i sintaksicheskogo analiza v sisteme Dialing. Tehnicheskaya documentacia (2000) (in russian), http://www.aot.ru/

5. Zolotova, G.A.: Sintaksicheskiy slovar: Repertuar elementarnykh edinits russkogo sintaksisa (in russian). Nauka, Moscow (1988) 